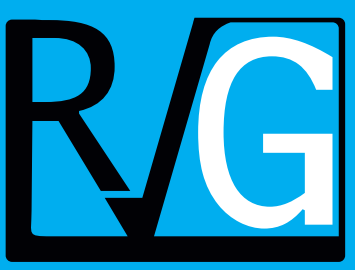

Año 21 No. 74

Abril - Junio 2016

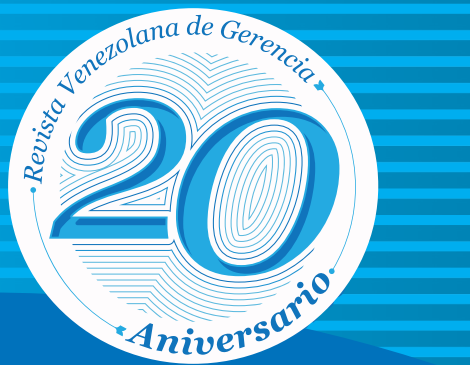

Venezolana de
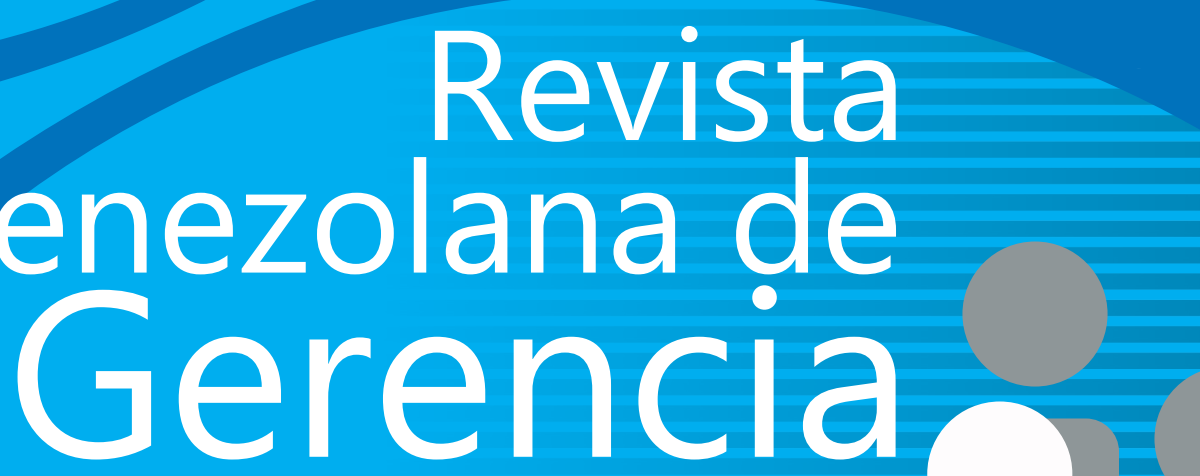


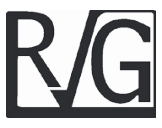

\title{
Departamentalización e innovación en las micro, pequeñas y medianas empresas de Colombia ${ }^{1}$
}

\author{
Gálvez-Albarracín, Edgar Julián ${ }^{2}$ \\ Hernández-Fernández, Lissette ${ }^{3}$ \\ Molina-Morejón, Víctor Manuel ${ }^{4}$
}

\section{Resumen}

La estructura organizacional es tema de alto interés académico por su relación con la estrategia y otros aspectos empresariales, por ello el objetivo de este trabajo es determinar en las micro, pequeñas, y medianas empresas de Colombia el impacto de la departamentalización sobre su innovación, específicamente en productos/servicios, procesos, gestión y globalmente. Para ello se realizó un estudio empírico de corte transversal e inferencial utilizándose regresiones lineales para procesar las respuestas de 1201 directivos de estas organizaciones, a quienes se consultó sobre el número de departamentos y la innovación en sus firmas. Los resultados muestran las organizaciones estudiadas en un nivel medio de departamentalización y confirman el impacto positivo de la especialización en la estructura sobre los tipos de innovación analizados (a excepción de procesos). Se concluye que la academia y las entidades de fomento deben apoyar a las empresas en su proceso de departamentalización equilibrando sus costos y beneficios.

Palabras clave: estructura organizacional; departamentalización; innovación; micro; pequeñas y medianas empresas.

Recibido: 10.08.15 Aceptado: 15.05.16

1 Este trabajo surge en el marco del proyecto de investigación "Análisis Estratégico para el Desarrollo de las MIPYMES en Iberoamérica de la Red Internacional de Investigadores en PYMES -FAEDPYME, en la que participan por Colombia las Universidades del Valle, Icesi, Santiago de Cali, Externado, La Sabana, Pontifica Bolivariana de Medellín, de la Costa (CUC), y Libre de Bogotá. Mayor información sobre la red ver el sitio www.faedpyme.upct.es

2 Profesor titular y coordinador del área académica de emprendimiento y pequeñas empresas, Facultad de Ciencias de la Administración, Universidad del Valle, Cali, Colombia. E-mail: edgar. galvez@correounivalle.edu.co

3 Profesora, Universidad de la Costa (CUC), Barranquilla, Colombia. E-mail: Ihernand31@cuc. edu.co

4 Profesor e investigador, Facultad de Contaduría y Administración, Universidad Autónoma de Coahuila, Torreón, México. E-mail: victormolina@uadec.edu.mx 


\title{
Departmentalization and innovation at micro, small and medium enterprises of Colombia
}

\begin{abstract}
The organizational structure is a subject of high academic interest because of its relationship with the strategy and other business aspects, so the aim of this work is to determine the impact of the departmentalization on innovation of micro, small, and medium enterprises in Colombia, specifically on products/services, processes, management and globally. To do that, an empirical, transversal and inferential study with linear regressions was conducted with 1201 managers of these organizations, who were asked about the number of departments and innovation in their firms. The results show the organizations studied in a medium level of departmentalization and confirm the positive impact of specialization in the structure on the different types of innovation analyzed (except for processes). It is concluded that the Academia and development agencies should support companies in their process of departmentalization, balancing costs and benefits.
\end{abstract}

Key words: organizational structure; departmentalization; innovation; competitiveness; MSMEs.

\section{Introducción}

Diferentes trabajos muestran la estructura organizacional como uno de los factores que pueden generar diferencias en el desempeño de las empresas, siendo una de las razones por las cuales la temática mantiene su vigencia investigativa (Marín y Campos, 2015; Piñeros et al, 2014; Martínez et al, 2013; McDonald y Marx, 2001). El interés en el asunto es naturalmente visible en el caso del Management que pasó de preocuparse simplemente por la especialización del trabajo y los procesos, a tener como uno de sus temas centrales la organización metódica y compleja de los recursos y las capacidades de la firma (Aktouf, 1998; Chiavenato, 1998).

Por otra parte la innovación como sinónimo de cambio, de nuevos productos y procesos es ampliamente reconocida y valorada en el desarrollo de empresas, sectores industriales y países (Naranjo y Calderón 2014; Santoreli, 2013; Escorsa Castells y Valls, 2003), ya que lleva a explotar con éxito las novedades en los ámbitos económico y social. Hoy la empresa está obligada a renovarse si quiere sobrevivir, si no pronto será alcanzada y dejada atrás por sus competidores debido al cada vez más corto ciclo de vida de los negocios (Medina y Espinosa, 1994).

Según King (1989) y Becker y Whisler (1967), existen variables externas e internas influenciadoras de la renovación empresarial, en este sentido Damanpour (1991) plantea que la generación, desarrollo e implantación de nuevas ideas y comportamientos depende de las características de la estructura organizativa. Así mismo Russell y Russell (1992) señalan que independientemente de la incertidumbre externa, la estructura organizacional debe proporcionar el contexto interno capaz de dirigir la ambigüedad y complejidad inherentes a la innovación, desde la concepción de los proyectos hasta su implementación.

Estudios como los de Damanpour (1991 y 1996), Kimberly y Evanisko 
Departamentalización e innovación en las micro, pequeñas y medianas empresas...

Edgar Gálvez-Albarracín, Lissette Hernández-Fernández, Víctor Molina-Morejón

(1981) y Daft (1978) muestran un impacto positivo de la especialización o nivel de departamentalización en la estructura de la organización sobre su desempeño innovador; sin embargo pocos han abordado el asunto en países emergentes y específicamente en sus micro, pequeñas y medianas empresas (MIPYMES) ${ }^{5}$.

En Colombia según las cifras del último censo económico realizado por el Departamento Nacional de Estadística (DANE), las MIPYME constituyen un $99,9 \%$ de las empresas, generan un $63 \%$ del empleo y un $37 \%$ de la producción (DANE, 2005). Por lo anterior trabajos como los de León et al, (2015), Mora et al, (2015), Gálvez et al, (2014), González y Hurtado, (2014) y Vera y Mora (2011) las han estudiado desde diferentes enfoques, continuando sin embargo pendiente por profundizarse el análisis de su estructura organizacional y las implicaciones de esta en sus diferentes resultados corporativos.

El presente trabajo busca contribuir a llenar los vacíos de conocimiento señalados respondiendo la siguiente pregunta: ¿Cuál es el impacto de la departamentalización sobre la innovación de MIPYMES de Colombia?, planteándose como objetivos específicos describirlo respectivamente en cuanto a productos/ servicios, procesos y gestión. Por estos motivos se realizó un estudio empírico de tipo inferencial y transversal con una muestra de 1201 MIPYMES de dicho país, utilizando regresiones lineales como método estadístico para contrastar las hipótesis planteadas.

\subsection{Estructura organizacional y departamentalización}

Sipara Chandler (1962) la estrategia determina el tipo de estructura requerida por la organización, para Damanpour (1987, 1991 y 1996) la complejidad motiva y justifica su especialización. En la literatura empresarial se define la complejidad como el número de actividades o subsistemas dentro de la organización, y según Zaltman et al, (1973) esta se puede abordar desde tres dimensiones: la profesionalización, la especialización y la diferenciación.

La profesionalización tiene en cuenta capacidades, conocimientos, intereses y motivaciones de los miembros de la organización para asignarles el mejor rol (Damanpour, 1991), la especialización los diferentes roles existentes en su interior (Hage y Aiken, 1967), y la diferenciación muestra su grado de división en unidades (Aiken et al, 1980; Kimberly y Evanisko, 1981; Damanpour, 1987, 1991 y 1996). Es así que por la complejidad de la actividad empresarial muchas firmas al desempeñar sus tareas requieren diversidad de especialistas y unidades (Slappendel, 1996).

Para referirse a la diferenciación los autores han utilizado denominaciones como diferenciación horizontal (Aiken et al, 1980), diferenciación estructural (Blau y Mckinley, 1979) y departamentalización (Young et al, 1982). Por su parte

5 En Colombia la Ley 590 (Congreso de la República, 2000) clasifica a las empresas por su tamaño según el número de trabajadores y el capital total así: 1) micro empresas: entre 1 y 10 trabajadores y un capital total hasta 500 salarios mensuales legales vigentes, 2) pequeñas empresas: entre $11 \mathrm{y}$ 50 trabajadores y un capital total entre 501 y 5000 salarios mensuales legales vigentes, y 3) mediana empresa: entre 51 y 200 trabajadores y un capital total entre 5001 y 30.000 salarios mensuales legales vigentes. 
Hellriegel y Slocum (1998) definen la departamentalización como la subdivisión de tareas y la asignación de estas a grupos especializados en una organización, así como a la creación de normas para el desempeño de esas tareas.

En relación con la supervisión, Bejarano (2006) señala la departamentalización como una buena opción de estructura organizacional pues generalmente la facilita, pero considera que cuando la empresa está sometida a fuertes cambios se debe pensar en trabajar por proyectos con lo cual se obtiene una mejor coordinación de los procesos, oficios, valores y creencias, administración y medición.

\subsection{Innovación}

La Organización para la Cooperación y el Desarrollo Económico (OECD) y la Oficina Europea de Estadística (EUROSTAT) señalan que la innovación es "la introducción de un nuevo, o significativamente mejorado producto (bien o servicio), de un proceso, de un nuevo método de comercialización o de un nuevo método organizativo en las prácticas internas de la empresa, la organización del lugar de trabajo o las relaciones externas" (OECD y EUROSTAT, 2005, p.56). En este sentido según la Comisión Europea (COM), la innovación permite integrar la tecnología existente para crear o mejorar un producto, un proceso o un sistema, logrando explotar con éxito la novedad en los ámbitos económico y social (COM, 2003).

Daft (1978), Han et al, (1998), Ravichandram (2000) y Sampere y Hervás (2014) destacan la necesidad de distinguir entre innovación técnica y administrativa, pues los estudios indican que las variables determinantes varían entre ellas (Swanson, 1994). La innovación técnica implica la generación de nuevos productos, servicios o procesos directamente relacionados con la actividad de trabajo principal y son distintas a las innovaciones que resultan meramente del uso de una tecnología (Knight, 1967; Daft, 1978; Kimberly y Evanisko, 1981; Damanpour y Evan, 1984; Damanpour, 1992).

Por su parte la innovación administrativa se manifiesta a través del cambio en las relaciones sociales, la comunicación, las reglas y las estructuras implicadas, inclusive la estructura y los procesos administrativos que están indirectamente relacionados con las actividades básicas de la organización y con su dirección (knight, 1967; Kimberly y Evanisko, 1981; Damanpour y Evan, 1984).

La Asociación Española de Contabilidad y Administración de Empresas (AECA) señala que todos estos conceptos en la organización se pueden integrar y sintetizar en tres tipos de innovación: -de productos/servicios, -de procesos y -de gestión (AECA, 1995); siendo el enfoque utilizado en este trabajo para analizar la innovación empresarial. La innovación en productos se materializa en la comercialización de nuevos artículos/ servicios o en la mejora de los existentes; en procesos en la dotación de nuevos equipos o procesos de producción; y en gestión en cambios o mejoras en la dirección, las compras, la comercialización y/o ventas entre otras.

\subsection{Estructura organizacional e innovación}

Damanpour y Gopalarishnan (1998), señalan que los teóricos abordan la relación entre la estructura y la innovación 
Departamentalización e innovación en las micro, pequeñas y medianas empresas... Edgar Gálvez-Albarracín, Lissette Hernández-Fernández, Víctor Molina-Morejón

desde diferentes enfoques: el tipo de innovación (técnica o administrativa) (Daft, 1978; Kimberly y Evanisko, 1981; Zmud, 1982), el nivel de radicalidad de la innovación (radical o incremental) (Dewar y Dutton, 1986; Nord y Tucker, 1987) y las fases de la innovación (Duncan, 1976; Zmud, 1982).

Según Ruiz (2003), la centralización en las decisiones influye negativamente en la innovación mientras que la flexibilidad y la baja formalización facilitan las nuevas ideas y comportamientos, aun así la diferenciación funcional de la empresa con su respectiva diversidad de especialistas implica un mayor conocimiento e incrementa la fertilización de ideas.

Es de esperar que una mayor variedad de especialistas proporcione acceso a una base más amplia de conocimientos (Kimberly y Evanisko, 1981), incremente la fecundación cruzada de ideas (Aiken y Hage, 1971) y facilite el reconocimiento de oportunidades para la innovación (Kim, 1980). Además, la diversidad de perspectivas y opiniones que surgen de los especialistas crea múltiples grupos de interés y demandas que estimulan la adopción de innovaciones (Kimberly y Evanisko, 1981; Wilson, 1965). Sin embargo la complejidad estructural incrementa los problemas de coordinación y control lo que lleva a una continua búsqueda de soluciones y propuestas de innovación.

Existen excepciones a la exaltación a la relación positiva entre la complejización de la estructura y la innovación (Daft y Becker, 1978; Blau y Mckinley, 1979; Russell, 1990; Russell y Russell, 1992), pues pueden presentarse problemas de conflicto de intereses e integración entre los distintos especialistas. La integración a través del contacto personal es un requisito necesario para el éxito de la innovación
(Lawrence y Lorsch, 1967), por tanto una excesiva segmentación impide la innovación debido a su influencia negativa en la integración (Kanter, 1985). Esto significa que la complejidad aumenta las propuestas de cambio pero la adopción solamente ocurrirá cuando existan suficientes mecanismos de integración para conseguir un buen grado de colaboraciónn (Zaltman et al, 1973).

Algunos autores señalan la diferenciación como generadora de innovación y otros conceptuan que no es generalizable pues depende del tipo de innovación (Evan y Black, 1967; Knight, 1967; Rowe y Boise, 1974; Downs y Mohr, 1976; Aiken et al, 1980; Kimberly y Evanisko, 1981; Zmud, 1982 y 1984; Damanpour, 1987; Damanpour et al, 1989; Han et al, 1998; Ravichandran, 2000; Darroch y McNaughton, 2002).

\subsection{Planteamiento de hipótesis}

Partiendo del fundamento teóricoconceptual previamente presentado y en coherencia con los estudios que se señalan más adelante, para cumplir con el objetivo de este trabajo se plantearon las siguientes hipótesis:

H1: El nivel de departamentalización de las MIPYME influye positivamente en la innovación de sus productos y/o servicios.

H2: El nivel de departamentalización de las MIPYME influye positivamente en la innovación de sus procesos.

H3: El nivel de departamentalización de las MIPYME influye positivamente en la innovación en su gestión.

H4: El nivel de departamentalización de las MIPYME influye positivamente en su innovación global. 
El planteamiento de las hipótesis $\mathrm{H} 1$ y $\mathrm{H} 2$ hace referencia a la innovación técnica, y la $\mathrm{H} 3$ a la innovación administrativa.

\subsection{Estudios previos sobre estructura organizacional, departamentalización e innovación empresarial}

Entre los trabajos que han abordado empíricamente estos factores de manera independiente o relacionándolos, por lo cual aportan antecedentes útiles para esta investigación pueden señalarse los siguientes:

Aiken y Hage encuentran en un trabajo presentadoen 1971 que unaelevada profesionalización y el mantenimiento de afiliaciones externas a la organización contribuyen a incrementar el potencial de innovaciones organizacionales.

En un estudio con organizaciones manufactureras de países en vías de desarrollo, Kim (1980) verificó una relación positiva entre la complejización de la estructura organizacional y la adopción de innovaciones técnicas. En la misma línea fueron los resultados de Kimberly y Evanisko (1981), quienes comprobaron que la especialización y la diferenciación funcional (departamentalización) influían en la adopción de innovaciones tecnológicas en los hospitales, aunque estas variables estructurales no eran determinantes para las innovaciones administrativas.

Dewar y Dutton (1986) y Ettlie et al, (1984) encuentran en sus trabajos que la concentración de especialistas crea una base de conocimiento motivadora de la innovación. Por su parte Damanpour (1996) analizando los factores determinantes de la estructura organizacional, el tamaño de la empresa y el efecto de ambos sobre la innovación en entornos inciertos, observan a la especialización de la estructura influyendo positivamente en la innovación técnica, especialmente en las empresas más pequeñas.

García (2009), encuentra que un $48,8 \%$ de las MIPYMES de Coahuila (México) no cuentan con departamento de comercialización, un $88,4 \%$ no dispone de uno de recursos humanos, un $95,1 \%$ no posee de investigación y desarrollo (I+D), un 60,6\% no tiene departamento de producción y un $54,4 \%$ no tiene de finanzas/contabilidad; en general estas empresas disponen en promedio de un sólo departamento.

Los resultados de la investigación realizada en Colombia por Gálvez et al, (2014) en el marco de la Fundación Análisis Estratégico para el Desarrollo de las MIPYMES (FAEDPYME), señalan como 2 el número medio de departamentos en las micro empresas, de 4 en las pequeñas y en las medianas de 5 , verificando que cuanto más grande son las firmas formales más diferenciadas tienen su estructura.

\subsection{Población y conformación de la muestra}

La población objeto de este estudio fueron las MIPYMES de Colombia, de las cuales se tomó una muestra de 1201 buscando un margen de confianza del $95 \%$ y un error del $5 \%$. Los criterios de selección de las empresas fueron que estuvieran ubicadas una de las en principales regiones geográficas del país y pertenecieran a la industria, la construcción, el comercio o los servicios (Tablas 1 y 2 ).

El instrumento utilizado para recolectar la información fue una encuesta estructurada a partir de la literatura disponible, la cual se aplicó telefonicamente entre mayo y julio del 2012 a los propietarios y/o directivos de las empresas, identificados a través de las bases de datos de las cámaras de comercio respectivas. 
Departamentalización e innovación en las micro, pequeñas y medianas empresas...

Edgar Gálvez-Albarracín, Lissette Hernández-Fernández, Víctor Molina-Morejón

Tabla 1

Conformación de la muestra por tamaño de las empresas y sector de actividad

\begin{tabular}{ccccc}
\hline $\begin{array}{c}\text { Tamaño de la empresa } \\
\text { Sector de actividad }\end{array}$ & $\begin{array}{c}\text { Micro de } \\
\mathbf{1} \text { a 10 } \\
\text { trabajadores }\end{array}$ & $\begin{array}{c}\text { Pequeña de } \\
\mathbf{1 1} \text { a 50 } \\
\text { trabajadores }\end{array}$ & $\begin{array}{c}\text { Mediana } \\
\mathbf{5 1} \text { a 200 } \\
\text { trabajadores }\end{array}$ & $\begin{array}{c}\text { Número de } \\
\text { empresas }\end{array}$ \\
\hline Industria & 304 & 143 & 118 & $\mathbf{5 6 5}$ \\
Construcción & 49 & 27 & 25 & $\mathbf{1 0 1}$ \\
Comercio & 234 & 57 & 15 & $\mathbf{3 0 6}$ \\
Servicios & 142 & 56 & 31 & $\mathbf{2 2 9}$ \\
Total & $\mathbf{7 2 9}$ & $\mathbf{2 8 3}$ & $\mathbf{1 8 9}$ & $\mathbf{1 2 0 1}$ \\
\hline
\end{tabular}

Fuente: Elaboración propia a partir de las bases de datos de las cámaras de comercio de Armenia Bogotá, Bucaramanga, Cali, Manizales, Medellín, Pasto, Pereira y Popayán.

Tabla 2

Conformación de la muestra por tamaño de las empresas y región geográfica de ubicación

\begin{tabular}{ccccc}
\hline $\begin{array}{c}\text { Tamaño de la empresa } \\
\text { Región y ciudad }\end{array}$ & $\begin{array}{c}\text { Micro de } \\
\mathbf{1} \text { a } \mathbf{1 0} \\
\text { trabajadores }\end{array}$ & $\begin{array}{c}\text { Pequeña de } \\
\mathbf{1 1} \text { a 50 } \\
\text { trabajadores }\end{array}$ & $\begin{array}{c}\text { Mediana } \\
\mathbf{5 1} \text { a 200 } \\
\text { trabajadores }\end{array}$ & $\begin{array}{c}\text { Número de } \\
\text { empresas }\end{array}$ \\
\hline $\begin{array}{c}\text { Centro Oriente (Bogotá y } \\
\text { Bucaramanga) }\end{array}$ & 123 & 149 & 129 & $\mathbf{4 0 1}$ \\
$\begin{array}{c}\text { Sur Occidente } \\
\text { (Cali, Pasto y Popayán) }\end{array}$ & 315 & 65 & 20 & $\mathbf{4 0 0}$ \\
$\begin{array}{c}\text { Antioquia y Eje Cafetero } \\
\text { Armenia, Manizales, Medellín } \\
\text { y Pereira) }\end{array}$ & 291 & 69 & 40 & $\mathbf{4 0 0}$ \\
Total & $\mathbf{7 2 9}$ & $\mathbf{2 8 3}$ & $\mathbf{1 8 9}$ & $\mathbf{1 2 0 1}$ \\
\hline
\end{tabular}

Fuente: Elaboración propia a partir de las bases de datos de las cámaras de comercio de Armenia Bogotá, Bucaramanga, Cali, Manizales, Medellín, Pasto, Pereira y Popayán.

\subsection{Variable de departamentalización}

Para medir el grado de diferenciación interna o "departamentalización" se solicitó a los gerentes y/o propietarios encuestados que informaran con cuáles de los siguientes departamentos contaba su empresa: 1) comercialización y ventas, 2) recursos humanos, 3) Investigación, desarrollo e innovación $(I+D+i)$, 4) operaciones y compras, 5) administración y contabilidad y 6) calidad. Con la suma de las respuestas afirmativas obtenidas se construyó la variable "nivel de departamentalización" 
que puede tener un rango teórico entre 0 y 6 , y es la utilizada para contrastar las hipótesis señaladas previamente.

\subsection{Variable de innovación}

Para medir el grado de innovación de la empresa existen dos enfoques (Hughes, 2001), uno objetivo que lo mide a partir de datos cuantitativos, como número de patentes o datos específicos de la innovación en productos (cantidad de nuevos productos) o procesos (costos de inversión); y un enfoque subjetivo basado en la percepción del gerente o propietario de la empresa sobre su actividad innovadora. En el caso de la MIPYME resulta más apropiado el enfoque subjetivo dado que evita subestimar su actividad innovadora (Hughes, 2001).

Para estudiar este factor en la investigación se tuvo en cuenta los diferentes conceptos recopilados en la revisión de trabajos realizada y especialmente el de AECA (1995) en cuanto a que en la organización se pueden presentar tres tipos de innovación: -de productos/servicios, -de procesos y -de gestión. Esta misma clasificación ha sido usada en trabajos como los de Maldonado et al, (2009), Van Auken et al, (2008) y Naranjo et al, (2008).

Para medir el grado de innovación en productos, procesos y gestión se utilizó una medida multicriterio de varios ítems por cada tipo de innovación, con una escala Likert de cinco puntos $(1=$ grado de innovación poco importante a $5=$ grado de innovación muy importante).

La variable "innovación en productos" (Innprod) está compuesta por la media aritmética de dos ítems (1) cambios o mejoras en productos o servicios existentes, y (2) comercialización de nuevos productos. La variable "innovación en procesos" (Innproc) está compuesta por la media aritmética de dos ítems: (1) mejoras o cambios en los procesos productivos, y (2) adquisición de nuevos equipos. Y la variable "innovación en gestión" (Inngest) está compuesta por la media aritmética de tres ítems: (1) cambios o mejoras en gestión de dirección, (2) cambios o mejoras en compras y aprovisionamientos, y (3) comercialización y ventas.

Por último se calcula una variable denominada "innovación global" (Innglob) que corresponde a la media aritmética de los resultados por empresa de las anteriores tres formas de innovación señaladas. La Tabla 3 muestra las diferentes preguntas realizadas para calcular esta variable, así como los resultados del estadístico Alpha de Crombach cuyos resultados demuestran la validez de la escala utilizada.

\subsection{Modelo teórico utilizado}

El siguiente es el modelo teórico utilizado en las regresiones lineales aplicadas para contrastar las hipótesis del trabajo: $\mathbf{Y}_{\mathrm{i}}=\mathbf{b}_{0(1 . .4)}+\mathbf{b}_{1(1 \mathrm{a} .1 \mathrm{1d})}$ Depart + $\varepsilon_{\mathrm{i}(1 . .4)}$ donde la variable dependiente es $\boldsymbol{Y}_{i}$ que corresponde respectivamente a cada una de las formas de innovación

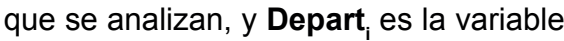
explicativa equivalente el nivel de departamentalización de las empresas.

\section{Impacto del nivel de departamentalización en la innovación de las micro, pequeñas y medianas empresas colombianas}

La Tabla 4 muestra los resultados descriptivos del estudio, se puede observar en las MIPYME un promedio de 3 departamentos, algunas que no cuentan con ninguno diferenciado y otras habiendo implementado los 6 consultados, siendo esta diferencia entendible por la diversidad del tamaño de las organizaciones abordadas. 
Departamentalización e innovación en las micro, pequeñas y medianas empresas... Edgar Gálvez-Albarracín, Lissette Hernández-Fernández, Víctor Molina-Morejón

\section{Tabla 3 \\ Variable de innovación}

\begin{tabular}{lll}
\hline & $\begin{array}{l}\text { Indique cuál ha sido el grado de innovación realizado en su } \\
\text { empresa en los dos últimos años: (1= Poco importante, 5= Muy } \\
\text { importante) }\end{array}$ & Validación escalas \\
$\begin{array}{l}\text { Innovación } \\
\text { en productos }\end{array}$ & $\begin{array}{l}\text { (1) Cambios o mejoras en productos o servicios existentes, y } \\
\text { (2) Comercialización de nuevos productos. }\end{array}$ & a de Cronbach = \\
& & 0,697 \\
Innovación & (1) Mejoras o cambios en los procesos productivos, y & a de Cronbach = \\
en procesos & (2) Adquisición de nuevos equipos & 0,679 \\
Innovación & (1) Gestión de dirección, & \\
en gestión & (2) Compras y aprovisionamientos, y & a de Cronbach $=$ \\
Innovación & (3) Comercialización y ventas & 0,737 \\
global & & $\alpha$ de Cronbach = 0,853 \\
\hline
\end{tabular}

Fuente: Elaboración propia.

Tabla 4

Descriptivos de las variables revisadas

\begin{tabular}{lllll}
\hline & Media & $\begin{array}{l}\text { Desviación } \\
\text { Estándar }\end{array}$ & Min & Max \\
\hline Grado de departamentalización & 3 & 2,01 & 0 & 6 \\
& & & & \\
Innovación: & 4,32 & 0,731 & 1 & 5 \\
En productos y/o servicios. & 4,29 & 0,727 & 1 & 5 \\
En procesos. & 4,16 & 0,788 & 1 & 5 \\
En gestión. & 4,26 & 0,701 & 1 & 5 \\
Innovación global. &
\end{tabular}

Fuente: Elaboración propia a partir de las bases de datos de las cámaras de comercio de Armenia Bogotá, Bucaramanga, Cali, Manizales, Medellín, Pasto, Pereira y Popayán.

En cuanto a la innovación puede verse la global en 4,26 señalando ello que los empresarios consideran sus organizaciones como bastante innovadoras y destacan su innovación de productos y/o servicios $(4,32)$, muy de cerca la de procesos $(4,29)$ y por último la de gestión $(4,16)$. Si bien estos resultados en general son muy positivos, inquieta ver los más bajos en la innovación de gestión, generándose así interrogantes para las facultades de administración de universidades e institutos de capacitación empresarial.

Por su parte la Tabla 5 muestra mayor departamentalización en las medianas empresas, seguidas de las pequeñas y por último en las micro; siendo en su orden los departamento más y menos implementados los de 
contabilidad/finanzas y los de investigación y desarrollo. Estos resultados denotan que los empresarios priorizan invertir sus recursos en asuntos obligatorios como las declaraciones de impuestos sobre otros como la diversificación de sus negocios.

\section{Tabla 5}

Existencia de departamentos según el tamaño de la empresa

\begin{tabular}{lllll}
\hline Tipo de departamento & Micro & Pequeña & Mediana & Sig. \\
\hline Comercialización/ventas. & $43,8 \%$ & $74,9 \%$ & $86,8 \%$ & $* * *$ \\
Recursos humanos. & $13,0 \%$ & $54,4 \%$ & $75,1 \%$ & $* * *$ \\
Investigación + desarrollo. & $11,4 \%$ & $25,4 \%$ & $39,7 \%$ & $* * *$ \\
Operaciones / compras. & $33,3 \%$ & $68,2 \%$ & $92,6 \%$ & $* * *$ \\
Contabilidad / finanzas. & $52,1 \%$ & $94,0 \%$ & $98,4 \%$ & $* * *$ \\
Calidad. & $37,2 \%$ & $61,1 \%$ & $81,5 \%$ & $* * *$ \\
\hline
\end{tabular}

Diferencias estadísticamente significativas: *al 90\% ; ** al 95\%; *** al 99\%.

Fuente: Elaboración propia a partir de las bases de datos de las cámaras de comercio de Armenia Bogotá, Bucaramanga, Cali, Manizales, Medellín, Pasto, Pereira y Popayán.

La tabla 6 muestra los resultados obtenidos mediante regresiones lineales por Mínimos Cuadrados Ordinarios (MCO) para conocer el impacto de la departamentalización sobre los cuatro tipos de innovación considerados.
Inicialmente se comprobó la normalidad de la distribución, la no presencia de multicolinealidad en los modelos, y el supuesto de independencia entre los residuos a través del estadístico DurvinWatson.

\section{Tabla 6}

\section{Impacto de la departamentalización en la innovación de las MIPYME}

\begin{tabular}{ccccc}
\hline $\begin{array}{c}\text { Variable } \\
\text { Dependiente } \\
\text { Variables } \\
\text { Independientes }\end{array}$ & $\begin{array}{c}\text { Producto } \\
\text { / servicio }\end{array}$ & Procesos & Gestión & Global \\
\hline $\begin{array}{c}\text { Departamentalización } \\
\beta\end{array}$ & $\mathbf{0 . 0 6 2}^{*}$ & $\mathbf{0 . 0 5 5}$ & $\mathbf{0 . 0 9 7 ^ { * * * }}$ & $\mathbf{0 . 0 8 3 ^ { * * * * }}$ \\
$\mathrm{t}$ & $(1.780)$ & $(1.603)$ & $(2.625)$ & $(2.616)$ \\
$\mathbf{R}^{2}$ Ajustado & 0.03 & 0.02 & 0.08 & 0.06 \\
Durvin-Watson & 1.822 & 1.901 & 1.901 & 1.821 \\
$\mathbf{F}$ & $3.169^{*}$ & 2.569 & $6.890^{* * *}$ & $6.843^{* * *}$ \\
VIF más alto & 1.000 & 1.000 & 1.000 & 1.000 \\
\hline
\end{tabular}

Debajo de los coeficientes estandarizados entre paréntesis se coloca el estadístico t-student.

Nivel de significancia: * al 90\%; **al 95\%; *** al 99\%

Fuente: Elaboración propia. 
Departamentalización e innovación en las micro, pequeñas y medianas empresas...

Edgar Gálvez-Albarracín, Lissette Hernández-Fernández, Víctor Molina-Morejón

Como puede observarse la departamentalización impacta positivamente sobre las formas de innovación observadas (a excepción de la de procesos), principalmente en la de gestión, luego en la global y en menor medida en la de producto/servicio. Al ser las $R^{2}$ ajustadas relativamente bajas los resultados de esta investigación pueden extrapolarse a la población manteniendo la debida prudencia. A continuación se presentan detalladamente los resultados de esta investigación y se discuten frente a los de trabajos anteriores:

\section{a) Impacto del nivel de departamentalización en la innovación de los productos y/o servicios de las MIPYME}

El beta positivo y significativo $\left(\beta=0.62^{*}\right)$ hallado para esta contrastación indica que la departamentalización de la empresa estimula en ella el lanzamiento de nuevos productos/servicios al mercado y/o mejoras en los existentes; por otra parte al ser la $F$ positiva $y$ significativa $\left(3.169^{*}\right)$ se comprueba la validez global del modelo utilizado. Estos resultados permiten aceptar la primera hipótesis planteada en el trabajo y confirman las conclusiones a las que llegaron las investigaciones de Kimberly y Evanisko (1981), Damanpour (1996), Mazanti y Zoboli (2009) y Pullen et al, (2009).

\section{b) Impacto del nivel de} departamentalización en la innovación de los procesos de las MIPYME

Para esta relación la investigación no encuentra un estadístico significativo, ello no permiten demostrar que la departamentalización produzca cambios o mejoras en los procesos productivos de la MIPYME y debe rechazarse entonces lasegundahipótesis planteada en el trabajo. Estos resultados contradicen los de Kimberly y Evanisko (1981) y Damanpour (1996), quienes verificaron que la departamentalización influye positivamente en los procesos productivos; esta divergencia podría explicarse en que en los países más desarrollados los departamentos de producción incluyen personal mejor preparado técnicamente.

\section{c) Impacto del nivel de departamentalización en la innovación de la gestión de las MIPYME}

El coeficiente de regresión
$\left(\beta=0.097^{* * *}\right)$ indica un impacto
positivo y muy significativo de la
departamentalización sobre la
innovación en gestión de estas
empresas, es decir en sus prácticas de dirección, compras y aprovisionamiento, comercialización y en ventas; así mismo se comprueba la validez global del modelo $\left(F=6.890^{* * *}\right)$. Estos resultados permiten aceptar la tercera hipótesis de la investigación y están en consonancia con los obtenidos por Kimberly y Evanisko (1981) y Damanpour (1996), mostrando importantes oportunidades para la innovación administrativa a partir de la especialización de la estructura organizacional.

\section{d) Impacto de la departamentalización en la innovación global de las MIPYME}

Se encuentra nuevamente un beta positivo y muy significativo $\left(\beta=0.083^{\star * *}\right)$, esto indica que la departamentalización 
contribuye a un mejor desempeño general de la innovación en la MIPYME y permite comprobar la cuarta hipótesis planteada en el trabajo; a la vez se confirman la validez global del modelo utilizado pues la $\mathrm{F}\left(6.843^{* * *}\right)$ es significativa. Estos resultados confirman los de Aiken y Hage (1971), Dewar y Dutton (1986) y Ettlie et al, (1984) y permiten sugerir a los micro empresarios seguir especializando y asignando por departamentos las funciones al interior de la firma.

\section{Conclusiones}

Esta investigación ha permitido encontrar que en Colombia las micro, pequeñas y medianas empresas tienen un nivel medio de diferenciación funcional, y al crecer como lo señala la teoría de la complejidad van incrementando su número de departamentos para poder asumir más y diferentes tareas en un entorno altamente diversificado. También muestra a los directivos considerándolas altamente innovadoras en sus diferentes actividades aunque un poco menos en su gestión, esto podría abordarse implementando departamentos de recursos humanos, uno de los menos frecuentes en ellas, buscando se ocupen de la formación en habilidades gerenciales y comerciales.

Otro aporte importante del trabajo es confirmar que la departamentalización impacta positivamente la mayoría de formas de innovación analizadas en la MIPYME y especialmente la de su gestión, área en la cual precisamente muestran más espacio y necesidad de mejora. Esto se constituye en una gran oportunidad para las facultades y escuelas de administración de Colombia y de la región con capacidad y deseo de abordar en dicho país el mercado de formación y asesoría especializada en temas de estructura organizacional para este tipo de empresas.

Probablemente los retos más relevantes en el proceso de especialización de las funciones en la MIPYME son lograr la debida fluidez y complementariedad entre los departamentos establecidos y optimizar la relación de la inversión requerida frente a los beneficios a obtener. Estos asuntos se constituyen en factores clave al pretender que los empresarios se interesen y acepten crear nuevas áreas funcionales en sus organizaciones.

$$
\text { Los hallazgos de esta }
$$

investigación tienen implicaciones para empresarios y gerentes porque les permite comprobar que invertir en departamentalizar sus organizaciones genera impactos positivos en la innovación de las mismas, y ello probablemente redundará en una mejor posición competitiva. A las entidades de fomento a las actividades económicas y a la académica les reitera la importancia de orientar a los empresarios para que cuando el crecimiento y naturaleza de sus actividades se lo demanden y permitan, especialicen la estructura de sus firmas.

Este trabajo presenta entre otras limitaciones el no haber incluido dentro de la muestra, empresas de la región norte de Colombia ya que constituyen un porcentaje importante de la demografía empresarial del país (esto fue debido a inconvenientes presupuestales para aplicar la encuesta en dicha región). Así mismo no haber entrevistado a funcionarios de segundo nivel jerárquico dentro de la empresa, lo cual podía haber reducido la probabilidad de que se presentaran sesgos en las respuestas relacionadas con el desempeño innovador. 
Departamentalización e innovación en las micro, pequeñas y medianas empresas... Edgar Gálvez-Albarracín, Lissette Hernández-Fernández, Víctor Molina-Morejón

Entre las futuras investigaciones que pueden derivarse de esta se encuentran: analizar la relación estudiada según el sector de actividad de la empresa y/o la región geográfica donde se ubica. También aplicar la prueba en la región norte del país para ver los resultados en dicha zona geográfica y cómo afecta el acumulado nacional; así mismo analizar independientemente el efecto de poseer cada tipo de departamento (recursos humanos, contabilidad y finanzas, etc.) sobre los diferentes tipos de innovación observados.

\section{Referencias bibliográficas}

Asociación Española de Contabilidad y Administración de Empresas AECA (1995), La innovación en la empresa: factor de supervivencia. Principios de organización y sistemas. Madrid: Asociación Española de Contabilidad y Administración de Empresas.

Aiken, Michael; Bacharach, Samuel y French, Lawrence (1980), Organizational structure, work process, and proposal making in administrative bureaucracies, Academy of Management Journal, Vol. 23, No. 4 pp. 631-652.

Aiken, Michael y Hage, Jerald (1971), The organic organization and innovation. Sociology, Vol. 5, No.1, pp. 63-82.

Aktouf, Omar (1998), La Administración: entre tradiciones y renovación. Cali-Colombia. Edit. Artes Gráficas. Universidad del Valle.

Becker, Slwyn y Whisler, Thomas (1967), The innovative organization: a selective view of current theory and research. Journal of Business, Vol. 40, No.4, pp. $462-469$

Bejarano, Rafael. (2006), Gestión de la innovación. Una visión actualizada para el contexto Iberoamericano. Cuba, Editorial Academia.

Blau, Judith y Mckinley, William (1979), Ideas, complexity and innovation. Administrative Science Quarterly, Vol. 24, No.2, pp. 200-219.
EscorsaCastell, Pere y Valls, Jaume (2003), Tecnología e innovación en la empresa: Dirección y gestión. Barcelona, España, Ediciones UPC.

Chandler, Alfred (1962), Strategy and structure: Chapters in the history of the American enterprise. Massachusetts Institute of Technology, Cambridge.

Chiavenato, Idalberto (1998), Introducción a la administración. México, Edit. McGraw-Hill.

Comisión Europea COM (2003), Comunicación de la Comisión al Consejo, al Parlamento Europeo, al Comité Económico y Social Europeo y al Comité de las Regiones. Bruselas, Bélgica.

Congreso de la República de Colombia (2000), Ley 590 de 2000. Diario oficial 44078. Colombia.

Daft, Richard (1978), A Dual-core model of organizational innovation. Academy of Management Journal, Vol. 21, No.2, pp. 193-210.

Daft, Richard y Becker, Selwyn (1978), The innovate organization. New York: Elsevier Press.

Damanpour, Fariborz (1987), The adoption of technological, administrative and ancillary innovations: impact of organizational factors. Journal of Management, Vol. 13, No.4, pp. 675688.

Damanpour, Fariborz (1991), Organizational innovation: a meta-analysis of effects of determinants and moderators. Academy of Management Journal, Vol. 34, No. 3, pp. 555-590

Damanpour, Fariborz (1992), Organizational size and innovation. Organizational Studies, Vol. 13, No.3, pp. 375-402.

Damanpour, Fariborz (1996), Organizational complexity and innovation: developing and testing multiple contingency models. Management Science, Vol. 42, No.5, pp. 693-716.

Damanpour, Fariborz y Evan, William (1984), Organizational innovation and performance: the problem of organizational lag. Administrative Science Quarterly, Vol. 29, No.3, pp. 392-409. 
Damanpour, Fariborz. y Gopalakrishnan, Shanti (1998), Theories of organizational structure and innovation adoption: the role of environmental change. Journal of Engineering and Technology Management, Vol. 15, No. 1, pp. 1-24.

Damanpour, Fariborz; Szabat, Kathryn y Evan, William (1989), The relationship between types of innovation and organizational performance. Journal of Management Studies, Vol. 26, No. 6, pp. 587-601.

Departamento Nacional de Estadísticas DANE (2005), Censo general 2005. Bogotá.

Recuperado el 07 de abril del 2016 de: http://www.dane.gov.co/censo/files/ libroCenso2005nacional.pdf

Darroch. Jenny y McNaughton, Rod (2002), Examining the link between knowledge management practices and types of innovation. Journal of Intellectual Capital, Vol. 3, No. 3, pp. 210-222.

Dewar, Robert y Dutton, Jane (1986), The adoption of radical and incremental innovations: An empirical analysis. Management Science, Vol. 32, No. 11 , pp. 1422-1433.

Downs, George y Mohr, Lawrance (1976), Conceptual issues in the study of innovation. Administrative Science Quarterly, Vol. 21, No.4, pp. 700-714.

Duncan, Robert (1976), The ambidextrous organization: designing dual structures for innovation. The management of organization, Vol. 1, pp. 167-188.

Ettlie, John; Bridges, William y O'keefe, Robert (1984), Organization strategy and structural differences for radical versus incremental innovation. Management Science, Vol. 30, No. 6, pp. 682-695.

Evan, William y Black, Guy (1967), Innovation in business organizations: some factors associated with success or failure. The Journal of Business, Vol. 40, No. 4, pp. 519-530.

Gálvez, Edgar; Cuellar, Karen; Restrepo, Carlos; Bernal, Cesar y Cortés, Juan (2014), Análisis estratégico para el desarrollo de las MIPYMES en Colombia. Editorial Universidad del Valle. Cali, Colombia.

Gálvez, Edgar; Riascos, Sandra y Contreras Fred. (2014), Influencia de las tecnologías de la información y comunicación en el rendimiento de las micro, pequeñas y medianas empresas colombianas. Estudios Gerenciales. Vol. 30, No. 133, pp. 355-364.

García, Domingo (2009), Análisis Estratégico para el Desarrollo de la Pequeña y Mediana Empresa del Estado de Coahuila (México). Editorial Universidad de Cantabria. España.

González, Carlos y Hurtado, Andrea (2014), Influencia de la capacidad de absorción sobre la innovación: un análisis empírico en las Mipymes colombianas. Estudios Gerenciales, Vol. 30, No.132,pp. 277-286.

Hage, Jerald y Aiken, Michel (1967), Relationship of centralization to other structural properties. Administrative Science Quarterly, Vol. 12, No. 1, pp. 72-91.

Han, Jin; Kim, Namwoon y Srivasta, Rajendra (1998), Market orientation and organizational performance: is innovation a missing link?. Journal of Marketing, Vol. 62, No. 4, pp. 30-45.

Hellriegel, Don y Slocum John (1998), Administración. Soluciones empresariales. México, Thomson Editores, Séptima Edición, 864p.

Huges, Alan (2001), Innovation and Business Performance: Small Entrepreneurial Firms in the UK and the EU. New Economy, Vol. 8, No. 3, pp. 157-163.

Kanter, Rosabeth (1985), Supporting innovation and venture development in established companies. Journal Bussiness Venturing, Vol. 1, No. 1, pp. 47-60.

Kim, Linsu (1980), Organizational innovation and structure. Journal of Business Research, Vol. 8, No. 2, pp. 225-245.

Kimberly, John y Evanisko, Michael (1981), Organizational innovation: the influence of individual, organizational, 
Departamentalización e innovación en las micro, pequeñas y medianas empresas...

Edgar Gálvez-Albarracín, Lissette Hernández-Fernández, Víctor Molina-Morejón

and contextual factors on hospital adoption of technological and administrative innovations. Academy of Management Journal, Vol. 24, No. 4, pp. 689-713.

King, Nigel (1989), Innovation in elderly care organizations: process and attitude. PhD Thesis, University of Sheffield, UK.

Knight, Kenneth (1967), A descriptive model of the intra firm innovation process. The Journal of business, Vol. 40, No. 4, pp. 478-496.

Lawrence, Paul y Lorsch, Jay (1967), Organization and environment, Managing differentiation and integration. Boston, Massachusetts: Harvard business School press.

León, Guillén; Castán, José y Afcha, Sergio (2015), Responsabilidad social en las MIPYMES de Sincelejo, Sucre. Dimensión empresarial, Vol. 12, No. 2, pp.185-203.

McDonald, Glen y Marx, Leslie (2001), Adverse specialization. Journal of Political Economy, Vol.109, No.4, pp.864-899.

Maldonado, Gonzalo; Madrid, Antonia; Martínez, María y Aguilera, Luis (2009), Los efectos de la innovación en el rendimiento de las MIPYMES de Aguascalientes: una evidencia empírica. Recuperado el 25 de julio del 2014 de: http://www.revista economia.uady.mx/2009/XXVI/73/02. pdf

Marín, D. A. y Campos, L. A. (2015), Estructura organizacional y relaciones inter-organizacionales: análisis en Instituciones Prestadoras de Servicios de Salud públicas de Colombia. Estudios Gerenciales, Vol. 31, No. 134, 88-99.

Martínez, Rafaela; Vera. María y Vera José (2013), La estructura organizacional en las pequeñas empresas constructuras de Puebla, México. Revista internacional de administración y finanzas, Vol. 6 , No.3, pp.71-83.

Mazzanti, Massimiliano y Zoboli, Roberto (2009), Embedding environmental innovation in local production systems: SME strategies, networking and industrial relations: evidence on innovation drivers in industrial districts. International Review of Applied Economics, Vol. 23, No. 2, pp. 169.

Medina, Cesar y Espinoza, Mónica (1994), La innovación en las organizaciones modernas. Gestión y Estrategia, Vol. 5, pp. 54-63.

Mora, Edwin; Vera, Mary y Megarejo Zuray (2015), Planificación estratégica y niveles de competitividad de las Mipymes del sector comercio en Bogotá. Estudios Gerenciales, Vol. 31, No. 134 , pp. $79-87$.

Naranjo, Julia y Calderón, Gregorio (2015), Construyendo una cultura de innovación. Una propuesta de transformación cultural. Estudios Gerenciales, Vol. 31, No.135,pp. 223-236.

Nord, Walter y Tucker, Sharon (1987), Implementing rutine and radical innovations. Lexington Books: Lexington, MA.

Organización para la Cooperación y Desarrollo Económico OCDE y Oficina Europea de Estadística EUROSTAT (2005), Manual de Oslo. Noruega: Organización para la Cooperación y Desarrollo Económico y Oficina de Estadística de las Comunidades Europeas.

Piñeros, Rafael; Castro, Andrés; Farfán, Dalsy y Nova, Camilo (2014), Descripción de la estructura organizacional del área de sostenibilidad del Helm Bank. Pensamiento y Gestión, No.37, pp.66-96.

Pullen, Annemien; Weerd-Nederhof, Petra; Groen, Aard; Song, Michel y Fisscher, Olaf (2009), Successful patterns of internal SME characteristics leading to high overall innovation performance. Creativity and Innovation Management, Vol. 18 No.3, pp. 209-223.

Ravichandran, T. (2000), Swiftness and intensity of administrative innovation adoption: an empirical study of TQM in information systems. Decision Sciences, Vol. 31, No.3, pp. 691-720. 
Rowe, Lloyd y Boise, William (1974), Organizational innovation: current research and evolving concepts. Public Administration Review, Vol. 34, No. 3, pp. 284-293.

Ruiz, Antonia (2003), Determinantes y consecuencias de la innovación organizacional: Una aproximación desde la perspectiva de la calidad total. Tesis doctoral. Universidad de Granada. Facultad de Ciencias Económicas y Empresariales. Departamento de Organización de Empresas. Granada. España.

Russell, Robert (1990), Innovations in organizations: toward an integrated model. Review of Business, Vol. 12, No. 2, pp. 19-26.

Russell, Robert y Russell, Craig (1992), An examination of the effects of organizational norms, organizational structure, and environmental uncertainty on entrepreneurial strategy. Journal of Management, Vol. 18, No. 4, pp. 639-356.

Santoleri, Pietro (2013), Diversity and Intensity of ICT use effects on product innovation: evidence from Chilean micro-data. Paper presented at UNU-MERIT conference on Micro Evidence, on Innovation and Development, Santiago de Chile, 7th8th of November 2013. Recuperado el 17 de enero del 2014 de:

http://www.merit.unu.edu/MEIDE/ papers/2013/PietroSantoleri.pdf

Sempere, Francisca y Hervás,José (2014), Innovación tecnológica y no tecnológica: efectos complementarios en la performance empresarial. Economía Industrial, No.391, pp.7176.

Slappendel, Carol (1996), Perspectives on innovation in organizations.
Organizations Studies, Vol. 17, No. 1, pp. 107-129.

Swanson, Burton (1994), Information systems innovation among organizations. Management Science, Vol. 40, No. 9, pp. 1069-1092.

Van Auken, Howard; Madrid, Antonia y García, Domingo (2008), Innovation and performance in Spanish manufacturing SMEs. International Journal of Entrepreneurship and Innovation Management, Vol. 8, No.1, pp.36-56.

Vera, Mary y Mora, Edwin (2011), Líneas de investigación en micro, pequeñas y medianas empresas. Revisión documental y desarrollo en Colombia. Tendencias, Vol 12, No.1, pp.213226.

Young, Robert; Hougland, John. y Shepard, James (1982), Innovation in open systems: a comparative study of banks. Sociology and Social Research, Vol. 65, No. 2, pp.177-193.

Wilson, James (1965), Innovation in organizations: notes towards a theory, en Thompson, J.D. (eds): Approaches to organizational design.

Zaltman, Gerald; Duncan, Robert y Holbeck, Johnny (1973), Innovations and organizations. London, John Wiley and Sons.

Zmud, Robert (1982), Diffusion of modern software practices: influence of centralization and formalization. Management Science, Vol. 28, No.2 pp. 1421- 1431.

Zmud, Robert (1984), A Management Science, examination of "push pull" theory applied to process innovation in knowledge work. Management science, Vol. 30, No.6, pp. 727-738. 


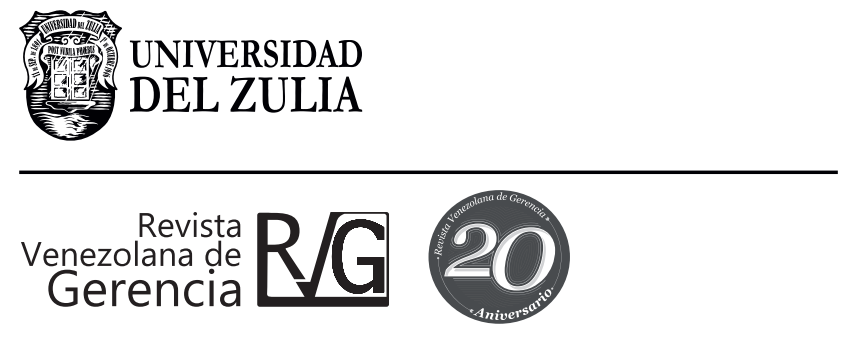

Año 21, No. 74

Esta revista fue editada en formato digital y publicada en junio del 2016, por la Universidad del Zulia, Vicerrectorado Académico, Serbiluz - Fondo editorial, Maracaibo -Venezuela. 